\title{
Verzeichnis der Tabellen, Schaubilder und Abbildungen
}

Tab. 1: $\quad$ Rentenbewegungen, Versicherungs- und Beitragsleistungen in der Angestelltenversicherung (1928 bis 1933) - 28

Tab. 2: $\quad$ Einnahmen der RfA 1926 bis 1933 (in Mio. RM) - 28

Tab. 3: $\quad$ Ausgaben der RfA für Pflichtleistungen sowie Heilfürsorge (in Mio. RM) 29

Tab. 4: $\quad$ Rentenbestand 1926 bis $1933-29$

Tab. 5: $\quad$ Rentenstruktur (Art der Leistungen und prozentualer Anteil bezogen auf die Gesamtzahl der Zugänge an Rentenanträgen) -29

Tab. 6: $\quad$ Entwicklung der Beitragsrückerstattungen an weibliche Versicherte wegen Heirat (1932 bis 1939) - 100

Tab. 7: $\quad$ Entwicklung der Berufungen gegen Rentenbescheide der RfA (1932 bis 1939) — 119

Tab. 8: $\quad$ Regionale Unterschiede in der Gehaltsklassenstruktur der RfA-Versicherten (Beitragsmarkenkauf im vierten Quartal 1939 in Stück und Anteil in Prozent — 294

Tab. 9: $\quad$ Vergleich der durchschnittlichen Angestellten-Monatsrenten im Altreich, in der „Ostmark“ (Österreich) und im Sudetenland im Jahr 1940 in RM — 294

Tab. 10: $\quad$ Entwicklung der Versicherten bei der RfA 1933 bis 1939 — 312

Tab. 11: Gesamteinnahmen der RfA 1933 bis 1939 (in Mio. RM) — 314

Tab. 12: $\quad$ Ausgaben der RfA für gesetzliche Pflichtleistungen (Renten und einmalige Zahlungen) und Heilverfahren 1933 bis 1939 (in Mio. RM) - 314

Tab. 13: $\quad$ Rentenbewegung 1933 bis $1939-314$

Tab. 14: Entwicklung der Durchschnittsrenten (Vollrenten) 1933 bis 1939 (in RM pro Monat) - 315

Tab. 15: Schichtung der laufenden Angestellten-Ruhegelder nach dem Stand vom 31. Mai $1937-316$

Tab. 16: $\quad$ Rentenbewegung 1939 bis $1944-437$

Tab. 17: Entwicklung der laufenden Renten im Altreich, in Österreich und dem Sudetenland (1939 bis 1944) - 479

Tab. 18: Entwicklung der durchschnittlichen Rentenhöhe im Vergleich von Altreich, Österreich und Sudetenland (1940 bis 1942) 479

Tab. 19: Übersicht über die zum Bestand des Altreichs der RfA hinzugekommenen aktiv Versicherten sowie Rentenempfänger in der Untersteiermark und in Kärnten (Stand Ende 1941) -497

Tab. 20: Übersicht über die regionale Verteilung der seit 1938 zum Bestand des Altreichs der RfA hinzugekommenen aktiv Versicherten sowie Rentenempfänger in den Westgebieten (Stand Ende 1941) - 530

Tab. 21: Übersicht über die regionale Verteilung der seit 1938 zum Bestand des Altreichs der RfA hinzugekommenen aktiv Versicherten sowie Rentenempfänger in den Ostgebieten (Stand Ende 1941) - 542

Tab. 22: Entwicklung der durchschnittlichen Rentenhöhe im Vergleich von Altreich, Österreich und Sudetenland sowie Ostoberschlesien/Polen (1940 bis 1942) - 562

Tab. 23: Zahl der RfA-Versicherten 1939 bis $1944-582$

Tab. 24: Gesamteinnahmen der RfA 1939 bis 1944/45 (in Mio. RM) - 583

Tab. 25: Ausgaben der RfA für gesetzliche Pflichtleistungen (Renten und einmalige Zahlungen) und Heilverfahren 1939 bis 1944 (in Mio. RM) — 585

Tab. 26: $\quad$ Entwicklung der laufenden Renten nach Regionen 1939 bis $1944-589$

Tab. 27: $\quad$ Entwicklung der Durchschnittsrente (Vollrenten) 1939 bis 1942 (in RM pro Monat) -590 
Tab. 28: $\quad$ Schichtung der laufenden Angestellten-Ruhegelder nach dem Stand vom 1.3. $1940-591$

Tab. 29: $\quad$ Vermögensentwicklung der RfA 1939 bis 1944/45 (in Mrd. RM) — 592

Schaubild 1: Organisation der Reichsversicherungsanstalt für Angestellte (RfA) $1931-32$

Schaubild 2: Organisation der Reichsversicherungsanstalt für Angestellte (RfA) $1937-170$

Schaubild 3: Organisation der Reichsversicherungsanstalt für Angestellte (RfA) $1942-376$

(In Klammer die jeweiligen Abbildungsnachweise)

Abb. 1: Bürobote (Bildarchiv der DRV-Bund, Berlin) - 34

Abb. 2: Das neue Kartei- und Archivgebäude der RfA in Berlin-Wilmersdorf, Ruhrstraße (Bildarchiv der DRV-Bund, Berlin) - 35

Abb. 3: Modernes RfA-Emblem 1930 (Verzeichnis der Gefolgschaftsmitglieder. Beilage zur Festschrift der RfA „25 Jahre Angestelltenversicherung 1913-1937“, Berlin 1937) — 36

Abb. 4: Protokoll der Sitzung des RfA-internen Ausschusses für die Durchführung des Gesetzes zur Wiederherstellung des Berufsbeamtentums vom 23. September 1933 (Privatarchiv Bonz) -48

Abb. 5: Das RfA-Zentralgebäude in der Ruhrstraße 2, Berlin Zehlendorf ca. 1935 (Bildarchiv der DRV-Bund, Berlin) - 53

Abb. 6: Der Beirat der RfA ca. 1936 (Bildarchiv der DRV-Bund, Berlin) -70

Abb. 7: RfA-Merkblatt „Lasst die Anwartschaft nicht verfallen“ von 1934 (RfA-Archiv Nr. 1) - 77

Abb. 8: Schreiben der Ingolstädter Vertrauensmänner an das RfA-Direktorium vom 15. Dezember 1933 (RfA-Archiv Fach 11, Nr. 9) - 80

Abb. 9: RfA-Merkblatt „Beitragserstattung bei Heirat“ von 1935 (RfA-Archiv Fach 53, Nr. 4) - 98

Abb. 10: „Richtlinien für die Rechtsberatungsstellen der DAF über die Art der Mitwirkung bei Durchführung des Angestelltenversicherungsgesetzes“ (Mitteilungen der RfA vom 26.1. 1935) 107

Abb. 11: Schreiben der DAF-Rechtsberatungsstelle Hamburg an die RfA vom 15. Februar 1938 (RfA-Archiv Nr. 86) - 117

Abb. 12: Programm zur Feier des 25-jährigen Bestehens der RfA am 17. Januar 1938 (Beilage zur Festschrift der RfA „25 Jahre Angestelltenversicherung 1913-1937“, Berlin 1937) — 146

Abb. 13: Brief eines Versicherten an Präsident Grießmeyer vom 8. August 1938 (RfA-Archiv Fach 98, Nr. 1) 148

Abb. 14: RfA-Präsident Albert Grießmeyer in Uniform 1939 (Bildarchiv der DRV-Bund, Berlin) 152

Abb. 15: Dienstschriftverkehr zwischen dem RAM und der RfA 1935 (RfA-Archiv Nr. 20) — 178

Abb. 16: Brief eines Versicherten an das RAM vom 20. Januar 1938 mit Kritik am Ausbaugesetz (RfA-Archiv Nr. 116, Nr. 8) - 188

Abb. 17: Die Anfrage des Sozialbüros der IG Farbenindustrie AG vom Juli 1939 (RfA-Archiv Nr. 91) 202

Abb. 18: Brief von Versicherten an die RfA vom 18. Dezember 1938 (RfA-Archiv Fach 11, Nr. 10) 204

Abb. 19: Brief eines Versicherten an die Direktion der RfA vom 20. Januar 1936 (RfA-Archiv Fach 108, Nr. 11) 207

Abb. 20: Rentendebatte im Berliner Lokal-Anzeiger vom Juni 1938 (RfA-Archiv Fach 111, Nr. 9) 209

Abb. 21: Versicherungskarte mit geklebten Beitragsmarken (RfA-Archiv o. Sign.) — 219

Abb. 22: Bericht eines Berliner Überwachungsbeamten vom 31. Dezember 1939 (RFA-Archiv Fach 104, Nr. 9) -222

Abb. 23: Schriftwechselkarte zu jüdischen Versicherten 1937 (RfA-Archiv o. Sign.) — 243 
Abb. 24: Bericht des Frankfurter Überwachungsbeamten vom 18. Mai 1939 zum Handwerkerversorgungsgesetz (RfA-Archiv Fach 110, Nr. 6) - 260

Abb. 25: Schreiben der RfA-Abwicklungsstelle Wien an Grießmeyer vom 16. Februar 1939 (RfA-Archiv Nr. 149) - 281

Abb. 26: Schreiben der RfA-Abwicklungsstelle Wien an die RfA Berlin vom 20. März 1939 (RfA-Archiv Nr. 146) -285

Abb. 27: Beispiel für eine Eingabe an Hitler mit der Beschwerde über Nachteile durch die Einführung der AV in Österreich vom März 1939 (BArch R 89/3493) — 292

Abb. 28: Eingabe des Reichsbundes der deutschen Kapital- und Kleinrentner, Gebiet Sudetenland, vom 14. Oktober 1939 an die RfA (RfA-Archiv Nr. 169 a) - 304

Abb. 29: Schreiben Grießmeyers an das RAM vom 13. September 1941 über die ungeplanten Auswirkungen des Leistungsverbesserungsgesetzes (BArch R 89/3171) - 333

Abb. 30: Schreiben Grießmeyers an das RAM vom 29. Oktober 1940 zum geplanten Altersversorgungswerk der DAF (BArch R 89/3173) - 342

Abb. 31: Briefe verunsicherter Beitragszahler an die RfA bezüglich des geplanten Altersversorgungswerks der DAF vom 14.2.1942 (RfA-Archiv Fach 119, Nr. 2) - 344

Abb. 32: Bericht der Überwachungsstelle Chemnitz vom 20. Juni 1941 zu den Auswirkungen der Presseveröffentlichungen zum Altersversorgungswerk (RfA-Archiv Fach 119, Nr. 2) - 346

Abb. 33: Präsidialverfügung Grießmeyers zur „soziale[n] Rechtsfindung“ vom 29.5 Mai 1942 (RfA-Archiv Fach 1, Nr. 3) 355

Abb. 34: Schreiben der Kreisleitung Rheingau an die RfA vom 12. August 1942 (RfA-Archiv Nr. 87) -356

Abb. 35: Schreiben der RfA vom 7. Februar 1944 betr. Rentenzahlung an eine jüdische Witwenrentenbezieherin (Privatarchiv Bonz) - 387

Abb. 36: Beschwerdeschreiben der DAF-Rechtsberatungsstelle Minden wegen Verzögerungen der Rentenfeststellung vom 25. August 1944 (BArch R 89/3414) - 408

Abb. 37: Bericht eines Überwachungsbeamten vom 21. Mai 1944 über die Verzögerungen bei der Rentenbearbeitung (RfA-Archiv Nr. 206 b) -410

Abb. 38: Die Beitragsklassengliederung ab 1. Oktober 1941 (RfA-Archiv Fach 12, Nr. 5) - 429

Abb. 39: Beschwerdebrief an die RfA betr. Beitragsrückerstattung an Frauen vom 30. Januar 1940 (RfA-Archiv Fach 97, Nr. 3) - 431

Abb. 40: Schreiben Grießmeyers an die Reichsministerien vom 8. Januar 1942 betr. Beitragsrückerstattung für Frauen (RfA-Archiv Fach 1, Nr. 1) — 433

Abb. 41: Schreiben des Regierungspräsidenten von Königsberg an Grießmeyer vom 11. Dezember 1940 betr. nicht erfüllter Anwartschaften von Gefallenen (RfA-Archiv Fach 113, Nr. 5) -441

Abb. 42: Interner RfA-Vermerk vom 12. Juli 1940 betr. Rentenansprüche verwundeter Soldaten (RfA-Archiv Nr. 7) -450

Abb. 43: Schreiben Grießmeyers an das RAM vom 5. Juli 1941 betr. Überweisung von Rentenleistungen an das Ausland (BArch R 89/3414) -460

Abb. 44: Dienstanweisung für die Leistungsabteilung vom 27. Januar 1941 betr. Einstellung von Rentenzahlungen an deportierte Juden (RfA-Archiv Regal 5, o. Signatur) - 461

Abb. 45: Schreiben der RfA an das RVA vom 25. Januar 1941 betr. Antrag auf Einstellung der Rentenzahlung wegen staatsfeindlicher Betätigung an einen inhaftierten jüdischen Ruhegeldempfänger (BArch R 89/3408) — 463

Abb. 46: Interne RfA-Vermerke vom 18. Dezember 1941 und 19. Januar 1942 betr. Rentenzahlungen an Juden (RfA-Archiv Nr. 198) -468

Abb. 47: Bittbrief eines jüdischen Versicherten an die RfA vom Juli 1941 (BArch R 89/3414) 471 
Abb. 48: Schreiben der RfA an das RAM betr. Rentenzahlungen an deportierte Juden in Litzmannstadt und Theresienstadt vom 22. November 1941 und vom 11. August 1942 (BArch R $89 / 3414)-472$

Abb. 49: Schreiben des Oberbürgermeisters von Leipzig an die RfA vom 12. Februar 1942 betr. Abnahme von Versicherungsunterlagen deportierter Juden (RfA-Archiv Fach 65, Nr. 6) -473

Abb. 50: Beschwerdebrief einer österreichischen Versicherten an die RfA vom 17. März 1940 (BArch R 89/3493) 484

Abb. 51: Schreiben der RfA-Abwicklungsstelle Wien an Grießmeyer vom 8. Mai 1944 (RfA-Archiv Nr. 158 a) 492

Abb. 52: Beschwerdebrief der NSDAP-Kreisleitung Gablonz an die RfA betr. Lage der sudetendeutschen Rentner vom 3. Januar 1940 (RfA-Archiv Nr. 172) — 500

Abb. 53: Schnellbrief des sudetendeutschen Reichsstatthalters Henlein betr. Regelung der Renten im Sudetenland vom 7. Dezember 1940 (RfA-Archiv Nr. 173) - 504

Abb. 54: Briefkopf der RfA, Amtsstelle Strassburg (RfA-Archiv Nr. 132) — 535

Abb. 55: Beschwerdebrief eines Versicherten vom 14. Oktober 1939 wegen des Volkstumsnachweises (RfA-Archiv Nr. 163) -548

Abb. 56: Handschriftlicher Vermerk von RfA-Direktor Koch vom 5. Dezember 1939 (RfA-Archiv Nr. 163) - 553

Abb. 57: Schnellbrief des RAM an den Regierungspräsidenten in Kattowitz vom 1. April 1940 betr. Einführung der Rentenversicherung in den angegliederten polnischen Gebieten (RfA-Archiv Nr. 163 a) -558

Abb. 58: Schreiben der RfA an Parteistellen wegen nicht anerkannter Nachweise der deutschen Volkszugehörigkeit vom Mai/Juni 1940 (RfA-Archiv Nr. 163 b) — 559

Abb. 59: Beschwerde der DAF-Rechtsberatungsstelle Kattowitz vom 8. Dezember 1941 über unzulängliche Renten (RFA-Archiv Nr. 163 b) -563

Abb. 60: Schreiben Grießmeyers vom 23. Dezember 1942 betr. Gewährung von Versicherungsleistungen an Juden in Oberschlesien (BArch R 89/3414) - 577

Abb. 61: Schreiben der RfA an das RVA vom 14. Oktober 1944 wegen Ruhens der Rente aufgrund staatsfeindlicher Tätigkeit (BArch R 89/23308) — 600

Abb. 62: Notiz eines RVA-Beamten vom 20. März 1945 betr. Rentenzahlung an einen KZ-Häftling (BArch R 89/23308) - 601 